\title{
Open Access für die Rechtswissenschaft: Pflicht oder Privatsache? - Perspektiven aus der akademischen Infrastruktur
}

Ivo Vogel"

Die dem Paradigma des Open Access folgende akademische Infrastruktur ist auf europäischer Ebene wie auch in Deutschland, Österreich sowie der Schweiz - je nach Fachrichtung - unterschiedlich ausgeprägt. Für die Rechtswissenschaft kann in den D-A-CH-Ländern jedoch noch keine feste oder etablierte akademische Infrastruktur im Hinblick auf Open Access konstatiert werden. Die vielfältigen Förderangebote werden durch die Rechtswissenschaft nur äußerst spärlich oder überhaupt nicht angenommen. Teilweise wird sich dem Open Access auch mit juristischen Mitteln entgegengestellt. Der nachfolgende Beitrag soll einen Überblick über die grundsätzlichen Fördermöglichkeiten und Infrastrukturen geben, die auch der Rechtswissenschaft zur Verfügung stehen, um den Open Access in dieser Disziplin zu befördern.

\section{A. Open Access-Förderung in Europa}

Einleitend soll zunächst kurz die europäische Perspektive beleuchtet werden. Die wohl jüngste Initiative auf der Ebene der Europäischen Union dürfte cOAlitionS ${ }^{1}$ sein. Am 4. September 2018 haben sich Science Europe sowie zunächst 11 nationale Forschungsförderorganisationen mit Unterstützung der Europäischen Kommission zum diesem Konsortium zusammengeschlossen. Gemäß der gemeinsamen Verpflichtungserklärung sollen nach dem 1. Januar 2021 Forschungsergebnisse aus öffentlichen Fördermitteln der beteiligten Forschungsförderungsorganisationen unmittelbar in Open Access-Zeitschriften oder auf Open Access-Plattformen veröffentlicht werden müssen. Dabei sind die Rahmenbedingungen für diese Initiative mittels zehn Punkten definiert (Plan S). Aus den D-A-CH-Ländern ist derzeitig nur Österreich mit dem FWF Wissenschaftsfonds beteiligt. Zwar hat sich auch die DFG positiv zu dieser Initiative geäußert, ${ }^{2}$ sie wird sich jedoch nicht einer Verpflichtung nach den Bedingungen von cOAlitionS und Plan S unterziehen, sondern vielmehr „künftig Fördermittelempfänger dazu auffordern, ihre Ergebnisse entweder auf dem sogenannten „Goldenen Weg“ (sofortige Open Access-Veröffentlichung) oder auf dem „Grünen Weg“ (Veröffentlichung in Open Access-Repositorien zusätzlich zur Veröffentlichung nach dem Subskriptionsmodell) öffentlich zugänglich zu machen. “3 und bleibt damit hinter der Verpflichtung der cOAlitionS

* BOR, Ass. iur. Ivo Vogel ist Leiter des Fachinformationsdienstes für internationale und interdisziplinäre Rechtsforschung (<intR $>^{2}$ an der Staatsbibliothek zu Berlin - Preußischer Kulturbesitz.Die Ausführungen in diesem Beitrag beruhen auf dem Informationsstand von Januar 2019.

1 https://www.coalition-s.org/.

2 https://www.dfg.de/foerderung/info_wissenschaft/2018/info_wissenschaft_18_56/.

3 Ebenda. 
zurück. Auch der SNF begrüßt diese Initiative zwar, erklärt jedoch die Zeitplanung mit der eigenen Open Access-Strategie für nicht kompatibel, lässt doch der SNF „die Erstveröffentlichung in einer kostenpflichtigen Zeitschrift und die nachträgliche Ablage auf einer OA-Plattform zu. Dieser [...] grüne Weg zu Open Access ist auch in der Nationalen Open Access-Strategie 2024 der Schweizer Hochschulen enthalten. ${ }^{4}$

Die Europäische Kommission und der Europäische Forschungsrat fördern und fordern schon seit längerer Zeit den Open Access, indem Projekte der entsprechenden Open Access-Policy unterworfen sind. Als beispielhaft gelten die Open AccessMandate im Rahmen von Horizon 2020 indem eine Open Access-Verpflichtung für die Verbreitung der Forschungsergebnisse in Form von Publikationen sowie der ihnen zugrundeliegenden Daten besteht. Publikationen und Forschungsdaten sind in elektronischer Form für Dritte unentgeltlich frei zugänglich zu machen, wobei die Kosten, die im Zusammenhang mit Open Access während der Projektlaufzeit anfallen und den allgemeinen Förderfähigkeitskriterien entsprechen (wie etwa $\mathrm{Pu}$ blikationsgebühren, Datenspeicherung etc.), in Horizon-2020-Projekten erstattungsfähig sind. Dabei ist sowohl der Grüne als auch der Goldene Weg wählbar und es bleibt den Projekten überlassen, welches Repositorium oder Online Archiv für die Veröffentlichung im Open Access genutzt wird. Als Best Practice gilt die Open Access-Infrastruktur OpenAIRE, ${ }^{5}$ die auf vorhandene Repositorien verlinkt sowie auf andere Register für Repositorien wie ROAR $^{6}$ oder Verzeichnisse wie OpenDOAR $^{7}$ verweist.

\section{B. Die nationale akademische Infrastruktur in Deutschland}

Als eines der Flaggschiffe der Open Access-Bewegung in Deutschland kann die 2008 von der Allianz der deutschen Wissenschaftsorganisationen gegründete Schwerpunktinitiative „Digitale Information“ bezeichnet werden, deren Ziel es ist, „Wissenschaftlerinnen und Wissenschaftler mit der bestmöglichen Informationsinfrastruktur auszustatten, die sie für ihre Forschung brauchen. " ${ }^{8}$ Im Hinblick auf acht definierte Handlungsfelder rund um die Digitalisierung in Wissenschaft und Forschung sollen die Aktivitäten und Ressourcen der Allianzpartner koordiniert und gebündelt werden, wobei die Allianz-Initiative für die Jahre 2018 bis 2020 ihre Arbeit, nachdem die Transformation von analogen Medien zu digitalen Objekten weit fortgeschritten ist, auf die genuin digitalen Publikationsformen und

4 https://oa100.snf.ch/de/news-de/freier-zugang-zu-publikationen-der-snf-unterstuetzt-den-europaeische n-plan-s/.

5 https://www.openaire.eu/.

$6 \mathrm{http}: / /$ roar.eprints.org/.

7 http://v 2.sherpa.ac.uk/opendoar/.

8 https://www.allianzinitiative.de/leitbild/. 
Strukturen konzentriert. Im Ergebnis sollen Querschnittsthemen wie Open Access, Open Science oder Forschungsdaten detailliert und strukturiert an die Wissenschaftscommunity adressiert werden. Den Open Access betreffend sind zwei Handlungsfelder „Wissenschaftliches Publikationssystem “9 und „Recht für Wissenschaft im digitalen Zeitalter" 10 besonders hervorzuheben.

Das im Handlungsfeld „Wissenschaftliches Publikationssystem“ „primär zu verfolgende Ziel ist die Ausschöpfung des Potentials digitalen Publizierens. Dies wird erreicht durch die offene Zugänglichkeit (Open Access) zu wissenschaftlichen Inhalten, die Erschließung des digital Möglichen sowie die nachhaltige Organisation des Publikationswesens als wissenschaftsfreundlicher Servicemarkt."11 Damit werden sowohl die Perspektiven der Nutzenden als auch der Verlage beleuchtet. Aktuell setzt sich diese Arbeitsgruppe intensiv mit der Verbindung von Open Access und Lizenzierung auseinander, wobei Themen von der Zeitschriftentransformation, über die Entwicklung neuer Geschäftsmodelle bis hin zur kooperativen Finanzierung bei Wahrung der Kostentransparenz und der Garantie der Nachhaltigkeit der Publikationsstrukturen behandelt werden. Bereits vorhandene Infrastrukturen wie institutionelle oder disziplinäre Open Access-Repositorien sollen dabei nicht in Vergessenheit geraten, leisten sie doch bereits jetzt einen wichtigen Beitrag zur Versorgung innerhalb von Forschungsinformationssystemen, indem sie Werkzeuge, Speicherplatz und Erschließungsstrukturen für die Veröffentlichung wissenschaftlicher Forschungsergebnisse im Open Access anbieten und - aufgrund der Weiterleitung in andere Suchsysteme - deren nationale und internationale Rezeption erheblich verbessern. Schließlich sind die Mitglieder dieser Arbeitsgruppe bestrebt, den kontinuierlichen Austausch auf der Policy-Ebene nachhaltig fortzusetzen und eine zielgenaue Lobbyarbeit zu leisten.

Die Mitglieder des Handlungsfelds „Recht für Wissenschaft im digitalen Zeitalter“ befassen sich mit den rechtlichen Dimensionen hinsichtlich digitaler Informationen und wie diese beispielsweise den Open Access beschränken oder ermöglichen. Im Zentrum stehen hier Themen des Urheber-, Leistungsschutz-, Datenschutz- und Persönlichkeitsrechts. Es bleibt abzuwarten, ob künftig auch das Wissenschaftsrecht (z.B. bezüglich der Änderung der Berufungspraxis) stärker in den Fokus dieses Handlungsfeldes rücken wird. Für die Periode 2018 bis 2020 liegen die Schwerpunkte eindeutig in der Lobbyarbeit.

9 https://www.allianzinitiative.de/handlungsfelder/wissenschaftliches-publikationssystem/.

10 https://www.allianzinitiative.de/handlungsfelder/recht-fuer-wissenschaft-im-digitalen-zeitalter/.

11 Ebenda. 
Im Ergebnis der Arbeit aller Arbeitsgruppen der Allianzinitiative wurden mit DEAL $^{12}$ und OA2020-DE ${ }^{13}$ bereits zwei wegweisende Projekte auf den Weg gebracht. Im Rahmen des Projekts DEAL ist die Hochschulrektorenkonferenz als Vertreterin der Allianz der Wissenschaftseinrichtungen damit beauftragt, mit den großen Wissenschaftsverlagen nationale Lizenzverträge für das gesamte Portfolio an elektronischen Zeitschriften abzuschließen und zugleich eine Open AccessKomponente zu etablieren.

Hingegen verfolgt der Nationale Open Access-Kontaktpunkt OA2020-DE das strategische Ziel, Voraussetzungen für die großflächige Open Access-Transformation zu schaffen, wobei hier die nationale Ebene im Vordergrund steht. Dieser Prozess wird durch umfängliche Publikations- und Kostendatenanalysen nationaler Hochschulen und Forschungseinrichtungen unterstützt, auf dessen Basis Open Access-Finanz- und Geschäftsmodelle entwickelt werden sollen. Ein erstes Pilotprojekt stellt die OPEN Library Politikwissenschaft ${ }^{14}$ dar. Hier wurde unter Vermittlung des Nationalen Open-Access-Kontaktpunkts zwischen dem Verlag transcript, dem Fachinformationsdienst (FID) Politikwissenschaft an der Staats- und Universitätsbibliothek Bremen sowie Knowledge Unlatched über ein Modell verhandelt, die Frontlist „Politikwissenschaft transcript 2019“ im Open Access zu veröffentlichen, wobei $50 \%$ der Kosten durch den FID und die restlichen 50\% durch die teilnehmenden Einrichtungen als Konsortium finanziert werden. Hiervon inspiriert verhandelte der Fachinformationsdienst für internationale und interdisziplinäre Rechtsforschung zusammen mit dem Peter Lang Verlag sowie Knowledge Unlatched ein ähnliches Modell für jährlich 10 Frontlist-Titel im IT-Recht und einer Laufzeit von 2019-2021.15

\section{Einzelakteure auf Bundesebene}

Lag die Schwerpunktförderung der Deutschen Forschungsgemeinschaft (DFG) im Bereich des Open Access in den vergangenen Jahren stark auf dem Gebiet der Finanzierung von Publikationsgebühren für die Veröffentlichung von Artikeln in Open Access-Zeitschriften, ${ }^{16}$ wovon nahezu alle Universitäten bereits Gebrauch gemacht haben, orientiert sich die DFG nunmehr mit dem Förderprogramm „Infrastruktur für elektronische Publikationen und digitale Wissenschaftskommunikation“ 17 stärker in Richtung der „Entwicklung komfortabler Dienstleistungen und Infrastrukturen für die Produktion, die Verbreitung und die Nutzung offen zu-

12 https://www.projekt-deal.de/.

13 https://oa2020-de.org/.

14 https://oa2020-de.org/pages/transcriptopenlibrarypowi/.

15 https://oa2020-de.org/pages/oaitlaw/

16 https://www.dfg.de/foerderung/programme/infrastruktur/lis/lis_foerderangebote/open_access/.

17 https://www.dfg.de/foerderung/programme/infrastruktur/lis/open_access/foerderung_open_access/in dex.html. 
gänglicher wissenschaftlicher Publikationen“ und wird damit den „geänderten Rahmenbedingungen der Wissenschaftskommunikation gerecht“.

Im Rahmen der DFG-Förderung sei das Förderformat der Fachinformationsdienste für die Wissenschaft herausgestellt. Aus den Sondersammelgebieten hervorgegangen und seit 2014 von der DFG gefördert sind diese angetreten, um für die jeweilige Wissenschaftscommunity bedarfsgerechte Services zur Fachinformation bereitzustellen bzw. weiterzuentwickeln. Ein zentraler Förderschwerpunkt ist dabei u. a. die Etablierung einer e-only bzw. e-prefered-Strategie. Auch wenn die Förderung des Open Access nicht explizit in den Richtlinien verlangt ist, verstärken die FID immer mehr ihre Aktivitäten in diesem Bereich vom Aufbau von Fachrepositorien über die Open Access-Beratung bis hin zur Erarbeitung von neuen Lizenzmodellen (FID-Lizenzen). Zur Seite steht den FID hierfür das Kompetenzzentrum für Lizenzierung (KfL), ${ }^{18}$ welches von der Niedersächsischen Staats- und Universitätsbibliothek Göttingen (Koordinationsstelle), der Staatsbibliothek zu Berlin, der Bayerischen Staatsbibliothek München und der Verbundzentrale des GBV betrieben wird. Besonders sei hier auf die Open Access-Klausel im Mustervertrag des KfL verwiesen, der eine entsprechende Publikation für die vertraglich vereinbarten Nutzerinnen und Nutzer der jeweiligen FID-Lizenz eröffnet. ${ }^{19}$

Erwähnung soll schließlich noch ein konkretes DFG-Projekt zur Förderung der Open Access-Transformation finden, das auf die Open Access-Komponenten der ebenfalls von der DFG geförderten Allianz-Lizenzen-Initiative beruht. DeepGreen $^{20}$ startete mit der Erkenntnis, dass die im Zusammenhang mit den AllianzLizenzen verhandelten Rechte der Autorinnen und Autoren, nach einer kurzen Embargofrist ihre in den lizenzierten Zeitschriften veröffentlichten Beiträge in der publizierten Form in ein frei wählbares institutionelles oder fachspezifisches Repositorium einstellen und öffentlich zugänglich machen dürfen, kaum bis nicht genutzt wird. DeepGreen wird mit der Unterstützung von Verlagen alle Artikel, für die entsprechende Open Access-Rechte gelten, einsammeln und den einzelnen Repositorien zuweisen.

Die Max-Planck-Gesellschaft fördert Open Access nicht erst seit der Unterzeichnung der Berliner Erklärung, zu deren Initiatoren und Erstunterzeichnern sie gehört. Die vielfältigen Aktivitäten reichen von der Open Access-Beratung, über das Angebot des Repositoriums MPG.PuRe (Möglichkeit, Publikationen nach dem sogenannten "Grünen Weg" des Open Access zu publizieren), bis hin zur zentralen Übernahme von Publikationskosten für Open Access-Zeitschriften durch den Abschluss von Rahmenverträgen mit verschiedenen Open Access-Verlagen und Ein-

18 https://www.fid-lizenzen.de/.

19 Vgl. $\$ 4$ des Musterlizenzvertrages https://www.fid-lizenzen.de/dateien/musterlizenz-2016-10-27.

20 https://deepgreen.kobv.de/de/deepgreen/. 
zelprojekten, die sich jedoch im Wesentlichen immer noch sehr stark auf den STMBereich konzentrieren. Für die Rechtswissenschaft gibt es nunmehr allerdings einen Lichtblick, haben doch der Verlag Nomos und die MPDL eine Rahmenvereinbarung $^{21}$ geschlossen, die es den Autorinnen und Autoren aller Max-Planck-Institute (nicht nur der juristischen) künftig ermöglicht, ihre Publikationen nicht nur in gedruckter Fassung sondern auch im Open Access zu veröffentlichen.

Keineswegs vergessen werden dürfen noch die Positionen der Fraunhofer-Gesellschaft, ${ }^{22}$ der Helmholtz-Gemeinschaft ${ }^{23}$ und der Leibniz-Gemeinschaft, ${ }^{24}$ die an dieser Stelle allerdings nicht näher ausgeführt werden können.

Im Bereich Digitalisierung in Bildung und Forschung wird Open Access ministeriell in Deutschland u. a. durch das Bundesministerium für Bildung und Forschung gefördert. Die im Jahr 2016 veröffentlichte Strategie ${ }^{25}$ gibt ein klares Bekenntnis zum Open Access als Grundprinzip der Wissenschaftsförderung in Deutschland ab. Flankiert wird diese Strategie durch die Veröffentlichung „Open-Access-Strategien für wissenschaftliche Einrichtungen - Bausteine und Beispiele" aus dem Jahre 2012, die den wissenschaftlichen Einrichtungen als Handreichung für die Umsetzung des Open Access dient. ${ }^{26}$

\section{Open Access-Aktivitäten auf Landesebene}

Die Bundesländer haben mittlerweile ihre eigene Infrastruktur und Strategien zur Open Access-Förderung aufgebaut, die unterschiedlich stark ausgeprägt sind. Vorreiter sind insbesondere die Länder Thüringen, ${ }^{27}$ Hamburg, ${ }^{28}$ Berlin, ${ }^{29}$ SchleswigHolstein ${ }^{30}$ sowie Baden-Württemberg. ${ }^{31}$ Baden-Württemberg ist aus dem Grunde ein besonderes Beispiel, als hier bereits der Gesetzgeber des Landes tätig geworden ist, wonach gemäß $\ 44$ Abs. 6 LHG BW die Hochschulen die Angehörigen ihres

21 https://www.mpdl.mpg.de/ueber-uns/nachrichten/396-max-planck-digital-library-kooperiert-mit-no mos.html.

22 https://www.fraunhofer.de/content/dam/zv/de/publikationen/fraunhofer-open-access-strategie-2020. pdf.

23 https://os.helmholtz.de/?id=802.

24 https://www.leibniz-gemeinschaft.de/fileadmin/user_upload/Bilder_und_Downloads/Forschung/Ope n_Science/Open_Access_Policy_web.pdf.

25 https://www.bmbf.de/pub/Open_Access_in_Deutschland.pdf.

26 https://www.bmbf.de/files/open-access-strategien.pdf.

27 https://www.thueringen.de/mam/th6/pub/digitalstrategie_2017.pdf.

28 https://www.buergerschaft-hh.de/ParlDok/dokument/59359/haushaltsplan-2017-2018-einzelplan-32-der-beh \%C3\%B6rde-f\%C3\%BCr-wissenschaft-forschung-und-gleichstellung-programm-hambur g-open-science-hos-.pdf.

29 http://www.open-access-berlin.de/.

30 https://www.schleswig-holstein.de/DE/Landesregierung/VIII/Presse/PI/PDF/2014/141118_msgwg_O penAccessStrategie.pdf?_blob=publicationFile\&v=3.

31 https://www.baden-wuerttemberg.de/fileadmin/redaktion/m-mwk/intern/dateien/Anlagen_PM/2014/ 066_PM_Anlage_E-Science_Web.pdf. 
wissenschaftlichen Personals durch Satzung verpflichten sollen, „das Recht auf nichtkommerzielle Zweitveröffentlichung nach einer Frist von einem Jahr nach Erstveröffentlichung für wissenschaftliche Beiträge wahrzunehmen, die im Rahmen der Dienstaufgaben entstanden und in einer periodisch mindestens zweimal jährlich erscheinenden Sammlung erschienen sind.“ Die Universität Konstanz hat das in einer entsprechenden Satzung umgesetzt, wogegen ihre Juristenfakultät Normenkontrollklage beim VGH Mannheim erhoben hat. Das Verfahren wurde ausgesetzt und an das Bundesverfassungsgericht verwiesen.

\section{E. Wer sonst noch beteiligt ist}

Eine besondere Stärkung hat das Open Access-Paradigma an den Universitäten durch die Gründung von Universitätsverlagen erfahren. In der Regel den Open Access-Leitlinien bzw. -Strategien der Universität folgend, werden überwiegend monographische oder Sammelwerke von Universitätsmitarbeitenden oder Absolventen größtenteils im Open Access veröffentlicht, da diese Verlage nicht gewinnorientiert agieren. Sie stellen eine sinnvolle Ergänzung zum kommerziellen Verlagswesen dar. Als Best Practice Beispiel für die Rechtswissenschaft ist der Universitätsverlag Göttingen ${ }^{32} \mathrm{zu}$ nennen, der auf 134 juristische Veröffentlichungen ${ }^{33}$ verweisen kann, womit das Recht auf Platz 1 des gesamten Fächerkanons der Universität Göttingen liegt. Verglichen mit der gesamten Buchproduktion ist das natürlich immer noch eine zu vernachlässigende Größe. Diese Bewertung verstärkt sich umso mehr, als im Directory of Open Access Books lediglich 544 Titel zum Recht nachgewiesen sind. ${ }^{34}$

Wissenschaft und Forschung werden zusätzlich auch durch öffentliche und private Stiftungen gefördert. Selbst in diesen Förderformaten halten Open Access-Mandate immer häufiger Einzug. Ein entsprechendes Bekenntnis gibt der Stifterverband mit seiner Initiative für offene Wissenschaft und Innovation ab. ${ }^{35} \mathrm{Im}$ Rahmen des Handlungsfelds Förderung der Initiative werden u. a. in Kooperation mit Wikimedia Deutschland und der Volkswagenstiftung die Fellowships Freies Wissen vergeben, die Doktoranden, Postdoktoranden, Juniorprofessoren oder wissenschaftliche Mitarbeitern, deren Hochschulabschluss nicht mehr als zehn Jahre zurückliegt, mit einem intensiven Mentoring- und Qualifizierungsangebot sowie 5.000 Euro fördert, um sie bei der öffentlichen Zugänglichmachung ihrer eigenen wissenschaftlichen Arbeit oder Projekte zu unterstützen. ${ }^{36}$ Mit dem Projekt Richter im Internet

32 https://www.univerlag.uni-goettingen.de/.

33 https://www.univerlag.uni-goettingen.de/handle/3/4.

$34 \mathrm{https}: / /$ www.doabooks.org/doab?func=subject\&cpId=46\&uiLanguage=en, hier sind die Rechtspublikationen des Universitätsverlages Göttingen enthalten.

35 https://www.stifterverband.org/initiative-offene-wissenschaft-innovation.

36 https://www.stifterverband.org/freies-wissen. 
hat davon auch bereits die Rechtswissenschaft profitiert, ist dies doch ein erster wichtiger Beitrag für die Open Data-Bereitstellung im Bereich der Justiz in Deutschland. ${ }^{37}$

Einen wesentlichen Beitrag zur Förderung des Open Access in Wissenschaft und Forschung in Deutschland steuern die Wissenschaftsblogs bei, sind die Blogbeiträge doch originär open und öffentlich zugänglich. Die ursprünglichen Vorbehalte gegen Blogs als unwissenschaftliche Meinungsäußerungen müssen zunehmend als veraltet gelten, da sich sowohl die Quantität als auch die Qualität wissenschaftlicher Blogbeiträge erheblich verbessert hat. Selbst renommierte Juristinnen und Juristen haben mittlerweile das Bloggen für sich entdeckt und erfahren eine enorme Rezeption ihrer wissenschaftlichen Erkenntnisse über das Internet und sprechen damit zunehmend den juristischen Nachwuchs an. Für die Rechtswissenschaft wären hier beispielhaft der Verfassungsblog, ${ }^{38}$ der Völkerrechtsblog ${ }^{39}$ und der Blog Junge Wissenschaft im Öffentlichen Recht (JuWissBlog) ${ }^{40}$ zu nennen.

Besonders für die (rechts)historische Forschung gelten retrodigitalisierte und mithin über das Internet frei zugängliche gemeinfreie Werke als wichtige Quelle für die Forschung. Verglichen mit den zuvor einzeln beschriebenen Strukturen, stellt die Retrodigitalisierung durch Bibliotheken und anderen Informationsinfrastrukturen quantitativ ein Schwergewicht dar. So lassen sich allein im Zentralen Verzeichnis für Digitalisierte Drucke ${ }^{41}$ 1.681.214 digitalisierte Werke finden, die der Wissenschaft (in unterschiedlicher Qualität) frei zur Verfügung stehen. Dabei ist die besondere Rolle von Bibliotheken hervorzuheben, die sich seit sehr langer Zeit mit finanziellen und personellen Ressourcen in diesen Bereich engagieren.

\section{F. Österreichs Open Access-Strukturen}

Österreich gehört zu den führenden europäischen Ländern im Bereich des Open Access und hat auf Initiative der Bundesregierung bereits früh eine nationale Open Innovation Strategie ${ }^{42}$ entwickelt. In diesem Kontext fördern hauptsächlich die Forschungsförderungsgesellschaft $(\mathrm{FFG})^{43}$ und Der Wissenschaftsfonds (FWF) auf Bundesebene den Open Access, wobei der der FWF Österreichs zentrale Einrichtung zur Förderung der Grundlagenforschung ist. Wie bereits erwähnt wurde, hat sich der FWF der europäischen Initiative cOAlitionS und dem Plan S angeschlos-

38 https://verfassungsblog.de/.

39 https://voelkerrechtsblog.org/.

40 https://www.juwiss.de/.

41 http://www.zvdd.de/startseite/.

42 http://openinnovation.gv.at/wp-content/uploads/2016/08/Open-Innovation-barrierefrei.pdf.

43 https://www.ffg.at/. 
sen, so dass dessen Forschungsförderung ab 2020 nunmehr nur noch bei Open Access-Veröffentlichung der Forschungsergebnisse erfolgt.

Dem ging 2015 eine Empfehlung der Arbeitsgruppe des Open Access Network Austria (OANA) ${ }^{44}$ voraus, bis 2025 die gesamte wissenschaftliche Publikationstätigkeit in Österreich auf Open Access umzustellen. Dieses Netzwerk wurde 2012 unter Beteiligung des FWF und der Österreichischen Universitätenkonferenz (UNIKO) gegründet und hat sich 2018, aufgrund der zunehmenden Vielfalt der im Netzwerk behandelten Themen in Open Science Network Austria umbenannt, ohne das alte (etablierte) Akronym zu ändern. Dem Kernteam von OANA gehören neben FFG, FWF und WWFT Universitäten und zahlreiche Forschungseinrichtungen an. In Arbeitsgruppen organisiert werden Empfehlungen zu Open Science erarbeitet, Ideen ausgetauscht und weitere Initiativen ins Leben gerufen. Die sich von Jahr zu Jahr ändernden Arbeitsschwerpunkte behandeln aktuell Open ScienceStrategie, ${ }^{45}$ Open Science-Information ${ }^{46}$ und die rechtlichen Aspekte von Open Science. ${ }^{47}$

Repräsentativ aber längst nicht abschließend seien neben OANA Projekte und Initiativen zum Open Access in Österreich wie die Austrian Open Science Support Group (AOSSG), Austrian Transition to Open Access (AT2OA), ${ }^{48}$ E-Infrastructures Austria plus ${ }^{49}$ oder das Netzwerk für RepositorienmanagerInnen (Repmannet ${ }^{50}$ genannt. Ähnlich wie das Projekt DEAL in Deutschland setzt sich die Kooperation E-Medien Österreich (KEMÖ) $)^{51}$ für die Förderung des Open Access in Österreich ein und stellt das eigene Bekenntnis zu diesem Paradigma durch den Abschluss von Open Access-Verträgen mit Wissenschaftsverlagen unter Beweis, ${ }^{52}$ die sich aber weitestgehend im Bereich der Naturwissenschaften bewegen.

\section{G. Die Rolle der Schweiz in der Open Access-Bewegung Europas}

Gemäß einer Studie der Europäische Kommission, die die bibliometrischen Daten der Datenbank Scopus zwischen 2009 und 2016 auswertet, ${ }^{53}$ belegt die Schweiz im internationalen Vergleich mit 39\% Open Access-Publikationen Platz 1, wobei weltweit hingegen nur knapp 30\% aller Forschungsergebnisse frei zugänglich sind.

\footnotetext{
44 https://www.oana.at/.

45 https://www.oana.at/arbeitsgruppen/ag-open-science-strategie/.

$46 \mathrm{https} / / / \mathrm{www}$.oana.at/arbeitsgruppen/ag-open-science-information/.

47 https://www.oana.at/arbeitsgruppen/ag-rechtliche-aspekte-von-open-science/.

48 https://at2oa.at/home.html.

49 https://www.e-infrastructures.at/de.

50 https://ubifo.wordpress.com/netzwerk-repositorienmanagerinnen-repmannet/.

51 https://www.konsortien.at/default.asp.

52 https://www.konsortien.at/openaccess.asp.

53 https:/ec.europa.eu/info/research-and-innovation/strategy/goals-research-and-innovation-policy/ope n-science/open-science-monitor/trends-open-access-publications_en\#open-access-to-publications.
} 
Einen wesentlichen Anteil an der Belegung dieser Spitzenposition hat natürlich der Schweizerische Nationalfond (SNF) ${ }^{54}$ mit seiner Open Access-Förderung. ${ }^{55}$

Die Veröffentlichung von Artikel in wissenschaftlichen Zeitschriften fördert der SNF für den goldenen Weg durch die Übernahme von Publikationsgebühren (APC), die direkt über die OA-Plattform auf mySNF ${ }^{56}$ beantragt werden können. Allerdings unterstützt er nicht das Publizieren nach dem sogenannten Hybrid-Modell, wobei aber die Open Access-Verpflichtung des SNF damit nicht ausgeschlossen wird. Für den Bereich der Monographien hat der SNF die Studie „OAPEN-CH - Auswirkungen von Open Access auf wissenschaftliche Monographien in der Schweiz" ${ }^{57}$ lanciert, die zu dem Ergebnis kommt, dass im Open Access herausgegebene digitale Ausgaben die Auffindbarkeit, die Sichtbarkeit und die Nutzung von Monographien erheblich verbessern, ohne dass sich dies negativ auf den Verkauf des gedruckten Buches auswirkt. ${ }^{58}$ Dies hat der SNF zum Anlass genommen, die Veröffentlichung von wissenschaftlichen Büchern im Open Access in Form der Übernahme der Book Processing Charge (BPC) zu fördern. ${ }^{59}$ Eine Förderung erfolgt unabhängig davon, ob diese aus einem SNF-finanzierten Projekt resultieren oder nicht.

Die Open Access-Politik des SNF fügt sich ein in die nationale Strategie der Hochschulen der Schweiz. Unter dem Dach der Rektorenkonferenz (swissuniversities) ${ }^{60}$ haben diese 2017 eine nationale Open Access-Strategie beschlossen, wonach alle mit öffentlichen Geldern finanzierten wissenschaftlichen Publikationen ab 2024 frei zugänglich sein sollen. ${ }^{61}$ Die im Aktionsplan zur Strategie vorgeschlagenen Maßnahmen reichen von der Einsetzung einer Governance und eines nationalen Monitorings zur Überwachung des Anteils an Open Access-Publikationen, über eine Kommunikations- und Sensibilisierungskampagne, die Bündelung von Dienstleistungen und Infrastrukturen (Einbeziehung kleinerer Hochschulen), bis hin zur rechtlichen Analyse im Hinblick auf Zweitveröffentlichung in der Schweiz sowie die Mitwirkung nationalen und internationalen Initiativen und Infrastrukturen. ${ }^{62}$ Schließlich wurde parallel hierzu ein Factsheet zur Verhandlungsstrategie von swissuniversities erarbeitet, das die Umgestaltung der nationalen Verträge über den

54 http://www.snf.ch/de/Seiten/default.aspx.

55 https://oa100.snf.ch/de/home-de/.

56 https://www.mysnf.ch/login.aspx? returnurl=\%2fdefault.aspx.

$57 \mathrm{http}: / /$ www.snf.ch/SiteCollectionDocuments/OAPEN-CH_schlussbericht_de.pdf.

$58 \mathrm{http} / / / \mathrm{www} . s n f . c h / d e /$ foerderung/wissenschaftskommunikation/oapen-ch/Seiten/default.aspx.

59 https://oa100.snf.ch/de/foerderung/buecher/.

60 https://www.swissuniversities.ch/de/.

61 https://www.swissuniversities.ch/fileadmin/swissuniversities/Dokumente/Hochschulpolitik/Open_Ac cess/Open_Access_strategy_final_DE.pdf.

62 https://www.swissuniversities.ch/fileadmin/swissuniversities/Dokumente/Hochschulpolitik/Open_Ac cess/Plan_d_action-d.pdf. 
Zugang zu wissenschaftlichen Zeitschriften mit den Großverlagen Springer Nature, Wiley und Elsevier mit Open Access-Komponenten zum Gegenstand hat ${ }^{63}$ und somit vergleichbar mit entsprechenden Projekten in Österreich und Deutschland ist.

\section{Fazit:}

Die zu Anfang beschriebene Zurückhaltung bis Ablehnung der Rechtswissenschaft in Bezug auf Open Access wird gerade in Anbetracht der bereits umfassend aufgebauten Infrastrukturen sowie der vielfältigen Fördermöglichkeiten in Deutschland, Österreich und der Schweiz umso deutlicher und immer weniger nachvollziehbar. Auch Juristinnen und Juristen sollten sich stärker des Paradigmas des Open Access annehmen, um einer notwendigen akademischen Geschlossenheit aller Wissenschaftszweige bezüglich dieses Themas ein weiteres Stück näher zu kommen. Es bleibt zu hoffen, dass die mit cOAlitionS auf den Weg gebrachte Selbstverpflichtung der nationalen Wissenschaftsfördereinrichtungen - als Bekenntnis zum Open Access - weitere Fürsprecher findet, welche dauerhaft die freie Zugänglichmachung zu wissenschaftlichen Forschungsergebnissen als Förderbedingung zur Pflicht erheben.

63 https://www.swissuniversities.ch/fileadmin/swissuniversities/Dokumente/Hochschulpolitik/Open_Ac cess/180315_Factsheets_Verhandlungsstrategie_D.pdf. 